\title{
ON GROWTH CHARACTERISTICS OF OPERATOR-VALUED FUNCTIONS
}

\author{
S.N. MISHIN
}

\begin{abstract}
In the work we generalize Liouville theorem and the concept of order and type of entire function to the case of an operator-valued function with values in the space $\operatorname{Lec}\left(\mathbf{H}_{1}, \mathbf{H}\right)$ of all linear continuous operators acting from a locally convex space $\mathbf{H}_{1}$ to a locally convex space $\mathbf{H}$ with an equicontinuous bornology. We find the formulae expressing the order and type of an operator-valued function in terms of the characteristics for the sequence of the coefficients. Some properties of the order and type of an operator-valued function are established.
\end{abstract}

Keywords: locally convex space, order and type of sequence of operators, order and type of entire function, equicontinuous bornology, convergence by bornology, operator-valued function.

\section{INTRODUCTION}

It is known [3, 4] that if an entire scalar function $f(z)=\sum_{n=0}^{\infty} a_{n} z^{n}$ is not a polynomial, the maximum of its modulus $M_{f}(r)=\max _{|z| \leq r}|f(z)|$ grows faster than any positive power of $r$ as $r \rightarrow \infty$ (Liouville theorem). To estimate the growth of such functions, one usually uses the characteristics (order and type),

$$
\rho=\varlimsup_{r \rightarrow \infty} \frac{\ln \ln M_{f}(r)}{\ln r}, \sigma=\varlimsup_{r \rightarrow \infty} \frac{\ln M_{f}(r)}{r^{\rho}} .
$$

At that, the formulae expressing these characteristics in terms of the coefficients

$$
\rho=\varlimsup_{n \rightarrow \infty} \frac{n \ln n}{-\ln \left|a_{n}\right|},(\rho e \sigma)^{\frac{1}{\rho}}=\varlimsup_{n \rightarrow \infty} n^{\frac{1}{\rho}} \sqrt[n]{\left|a_{n}\right|}
$$

are known. This work is devoted to the generalization of these formulae and the Liouville theorem for the case of an entire operator-valued function $F(t)=\sum_{n=0}^{\infty} A_{n} t^{n}$ with the values in the space $\operatorname{Lec}\left(\mathbf{H}_{1}, \mathbf{H}\right)$ of all linear continuous operators acting from a locally convex space $\mathbf{H}_{1}$ into a locally convex space $\mathbf{H}$. The spaces $\mathbf{H}_{1}$ and $\mathbf{H}$ are in general not normable.

\section{ENTIRE OPERATOR-VALUED FUNCTIONS AND ANALOGUE TO LIOUVILLE THEOREM}

$\mathbf{H}_{1}$ and $\mathbf{H}$ are separable locally convex spaces over the field of complex numbers with the topologies defined respectively by the multinorms $\left\{\|\cdot\|_{q}^{\prime}\right\}, q \in \mathcal{Q}$ and $\left\{\|\cdot\|_{p}\right\}, p \in \mathcal{P}$. Without loss of generality one can regard the multinorms in $\mathbf{H}_{1}$ and $\mathbf{H}$ as majorant [2]. By $\mathcal{A}=\left\{A_{n}\right\}_{n=0}^{\infty}$ we denote a sequence of linear continuous operators acting from the locally convex space $\mathbf{H}_{1}$

S.N. Mishin, On GROWTH CHARACTERISTICS OF OPERATOR-VALUED FUNCTIONS.

(C) Mishin S.N. 2013.

Submitted August 16, 2012. 
into the locally convex space $\mathbf{H}$. The sequence $\mathcal{A}$ is called as having an order [1, 5], if there exists a sequence of positive numbers $\left\{c_{n}\right\}_{n=0}^{\infty}$ such that

$$
\forall p \in \mathcal{P} \exists C_{p}>0 \exists q(p) \in \mathcal{Q} \forall x \in \mathbf{H}_{1} \forall n \in \mathbb{N}:\left\|c_{n} A_{n}(x)\right\|_{p} \leq C_{p}\|x\|_{q}^{\prime},
$$

i.e., the family of the operators $\left\{c_{n} A_{n}\right\}$ is equicontinuous.

Let

$$
\theta_{\mathcal{A}}(p, q, n)=\sup _{\|x\|_{q}^{\prime} \neq 0}\left\{\frac{\left\|A_{n}(x)\right\|_{p}}{\|x\|_{q}^{\prime}}\right\}, n=0,1,2, \cdots
$$

(the case $\theta_{\mathcal{A}}(p, q, n)=+\infty$ is not excluded). We denote

$$
\beta_{p, q}(\mathcal{A})=\varlimsup_{n \rightarrow \infty} \frac{\ln \theta_{\mathcal{A}}(p, q, n)}{n \ln n} .
$$

Definition 1. The number $\beta_{p}(\mathcal{A})=\inf _{q \in \mathcal{Q}} \beta_{p, q}(\mathcal{A}),(p \in \mathcal{P})$ is called a p-order of the sequence of the operators $\mathcal{A}$, and the number $\beta(\mathcal{A})=\sup _{p \in \mathcal{P}}\left\{\beta_{p}(\mathcal{A})\right\}$ is called its order.

If $\beta(\mathcal{A})= \pm \infty$ and at that the sequence $\mathcal{A}$ has an order, then it is called a sequence of an infinite order.

Remark. Let us note that there is an essential difference between the sequences having an order $\beta(\mathcal{A})=+\infty$, and that having no order (despite formally $\beta(\mathcal{A})=+\infty$ ). If $\beta(\mathcal{A})=+\infty$, but the sequence $\mathcal{A}=\left\{A_{n}\right\}$ has an order, it is possible to select a sequence of positive numbers $\left\{c_{n}\right\}$ such that condition (3) holds. And one can not select such a sequence for the sequences having no order.

If a sequence of operators $\mathcal{A}$ has a $p$-order $\beta_{p}(\mathcal{A}) \neq \pm \infty$, one introduces for it a finer characteristics. Denote

$$
\alpha_{p, q}(\mathcal{A})=\varlimsup_{n \rightarrow \infty} n^{-\beta_{p}(\mathcal{A})} \sqrt[n]{\theta_{\mathcal{A}}(p, q, n)} .
$$

Definition 2. The number $\alpha_{p}(\mathcal{A})=\inf _{q \in \mathcal{Q}} \alpha_{p, q}(\mathcal{A}),(p \in \mathcal{P})$ is called a $p$-type of a sequence of operators $\mathcal{A}$ at the p-order $\beta_{p}(\mathcal{A})$.

It is obvious that $\beta_{p}(\mathcal{A}) \leq \beta(\mathcal{A}), \forall p$. It is possible to show [7] that the case when the identity $\beta_{p}(\mathcal{A})=\beta(\mathcal{A})$ is valid not for all $p$, but just for some $p$, is reduced to the case $\beta_{p}(\mathcal{A})=\beta(\mathcal{A})$, $\forall p$ by replacing the multinorm to an equivalent one. This replacement changes neither the order no the type of a sequence of operators. This is why (without loss of generality) we shall consider two cases, either $\beta_{p}(\mathcal{A})=\beta(\mathcal{A}), \forall p$, or $\beta_{p}(\mathcal{A})<\beta(\mathcal{A}), \forall p$.

Definition 3. Let a sequence of operators $\mathcal{A}$ has the p-orders $\beta_{p}(\mathcal{A})$ and the order $\beta(\mathcal{A}) \neq$ $\pm \infty$. The number

$$
\alpha(\mathcal{A})=\left\{\begin{array}{cc}
\sup _{p \in \mathcal{P}}\left\{\alpha_{p}(\mathcal{A})\right\} & , \beta_{p}(\mathcal{A})=\beta(\mathcal{A}), \forall p \\
0 & , \quad \beta_{p}(\mathcal{A})<\beta(\mathcal{A}), \forall p
\end{array}\right.
$$

is called a type of the sequence of operators $\mathcal{A}$ at the order $\beta(\mathcal{A})$.

A sequence of operators $\mathcal{A}$ is called belonging to the class $\mathfrak{L}_{\mathbf{H}_{1}, \mathbf{H}}[b, a]$, (cf. [1, [5]) if its order is less than $b$ or equal to $b$, but then the type does not exceed $a$.

Let $\mathbf{H}$ be a complete space. It is known [8] that in this case the space $\operatorname{Lec}\left(\mathbf{H}_{1}, \mathbf{H}\right)$ of linear continuous operators acting from $\mathbf{H}_{1}$ into $\mathbf{H}$ equipped with an equicontinuous bornology is a complete bornological vector convex space.

Definition 4. An operator-valued function $F: \mathbb{C} \rightarrow \operatorname{Lec}\left(\mathbf{H}_{1}, \mathbf{H}\right)$ is called differentiable at a point $t_{0} \in \mathbb{C}$ if there exists a limit (w.r.t. the bornology of the space $\operatorname{Lec}\left(\mathbf{H}_{1}, \mathbf{H}\right)$ )

$$
\lim _{t \rightarrow t_{0}} \frac{F(t)-F\left(t_{0}\right)}{t-t_{0}}
$$


This limit is called a derivative of the operator-valued function $F$ at the point $t_{0}$ and is indicated by $F^{\prime}\left(t_{0}\right)$.

Definition 5. An operator-valued function $F: \mathbb{C} \rightarrow \operatorname{Lec}\left(\mathbf{H}_{1}, \mathbf{H}\right)$ is called entire if its defined and differentiable at each point $t \in \mathbb{C}$.

An entire operator-valued function is obviously continuous everywhere (w.r.t. the bornology of the space $\left.\operatorname{Lec}\left(\mathbf{H}_{1}, \mathbf{H}\right)\right)$.

Let

$$
\theta_{F}(p, q, t)=\sup _{\|x\|_{q}^{\prime} \neq 0}\left\{\frac{\|F(t)(x)\|_{p}}{\|x\|_{q}^{\prime}}\right\}, t \in \mathbb{C}
$$

(the case $\theta_{F}(p, q, t)=+\infty$ is not excluded).

Theorem 1. An entire operator-valued function $F(t)$ is bounded on each closed disk, i.e., the family of the operators $\{F(t)\}_{|t| \leq r}$ is equicontinuous for each $r>0$.

Proof. We fix an arbitrary $r>0$. Suppose the function $F(t)$ is entire, and the family $\{F(t)\}_{|t| \leq r}$ is not equicontinuous, i.e., there exists $p_{0} \in \mathcal{P}$ such that for each $C>0$ and for each $q \in \mathcal{Q}$ there exists $t_{C}=t_{C}(q)$ such that $\left|t_{C}\right| \leq r$ and $\theta_{F}\left(p_{0}, q, t_{C}\right)>C$. We fix an arbitrary $q \in \mathcal{Q}$ and take $C=n, n \in \mathbb{N}$. We obtain then a sequence of complex numbers $t_{n}=t_{n}(q)$ lying within the disk $|t| \leq r$. At that,

$$
\theta_{F}\left(p_{0}, q, t_{n}\right)>n, \forall n .
$$

By the boundedness of the sequence $\left\{t_{n}\right\}$ there exists a converging subsequence $\left\{t_{n_{k}}\right\}$. It follows from (5) that $\theta_{F}\left(p_{0}, q, t_{n_{k}}\right)>n_{k}, \forall k$, i.e., the sequence $\left\{F\left(t_{n_{k}}\right)\right\}$ is not equicontinuous and thus diverges. But by the continuity of the function $F$ it must converges. We obtain the contradiction.

If the function $F(t)$ is entire, then for each fixed $x \in \mathbf{H}_{1}, F(t)(x)$ is an entire function with values in $\mathbf{H}$. Such function is represented as a power series [9]

$$
F(t)(x)=\sum_{n=0}^{\infty} x_{n} t^{n}, x \in \mathbf{H}_{1},\left\{x_{n}\right\} \subset \mathbf{H}
$$

(the sequence $\left\{x_{n}\right\}$ depends on $x$ ). We let

$$
M_{F}(p, q, r)=\sup _{|t| \leq r} \theta_{F}(p, q, t) .
$$

We define a sequence of operators $A_{n}: \mathbf{H}_{1} \rightarrow \mathbf{H}$ as follows, $A_{n}(x)=x_{n}, \forall x \in \mathbf{H}_{1}$. We obtain the expansion of the function $F(t)$ as a power series

$$
F(t)=\sum_{n=0}^{\infty} A_{n} t^{n} .
$$

At that, series (6) everywhere pointwise converges to the function $F(t)$ (for each fixed $x \in \mathbf{H}_{1}$ the series $\sum_{n=0}^{\infty} A_{n}(x) t^{n}$ converges to the function $F(t)(x)$ everywhere). Let us show that $\left\{A_{n}\right\} \subset \operatorname{Lec}\left(\mathbf{H}_{1}, \mathbf{H}\right)$ and series (6) converges everywhere to the function $F(t)$ w.r.t. the bornology. First we prove the following theorem.

Theorem 2 (Analogue of Cauchy inequalty). The inequality

$$
\theta_{\mathcal{A}}(p, q, n) \leq \frac{M_{F}(p, q, r)}{r^{n}}, \forall p \forall q \forall n \forall r>0
$$

holds true. 
Proof. Let $p \in \mathcal{P}, q \in \mathcal{Q}, r>0$. If $M_{F}(p, q, r)=\infty$, then inequality (7) holds true. Let $M_{F}(p, q, r)<\infty$. Since for each fixed $x$ the vector-function $F(t)(x)=\sum_{n=0}^{\infty} A_{n}(x) t^{n}$ is entire, then (see, for instance, [9])

$$
A_{n}(x)=\frac{1}{2 \pi i} \int_{|\xi|=r} \frac{F(\xi)(x) d \xi}{\xi^{n+1}}, n \in \mathbb{N} .
$$

Hence, $\forall p \in \mathcal{P} \forall x \in \mathbf{H}_{1} \forall r>0 \forall n \in \mathbb{N}$ we have

$$
\left\|A_{n}(x)\right\|_{p} \leq \frac{\sup _{|\xi| \leq r}\|F(\xi)(x)\|_{p}}{r^{n}} \leq \frac{\sup _{|\xi| \leq r} \theta_{F}(p, q, \xi)}{r^{n}}\|x\|_{q}^{\prime}=\frac{M_{F}(p, q, r)}{r^{n}}\|x\|_{q}^{\prime}
$$

that yields inequality (7).

Since the function $F(t)$ is entire, by Theorem 1 for each $r>0$ the family $\{F(t)\}_{|t| \leq r}$ is equicontinuous, i.e.,

$$
\forall p \in \mathcal{P} \exists C_{p}>0 \exists q_{p} \in \mathcal{Q} \forall x \in \mathbf{H}_{1} \forall t|t| \leq r \Rightarrow\|F(t)(x)\|_{p} \leq C_{p}\|x\|_{q_{p}}^{\prime} .
$$

For each $p$ we choose $q_{0}=q_{0}(p)$ such that $\|x\|_{q_{0}}^{\prime} \geq\|x\|_{q_{p}}^{\prime}, \forall x \in \mathbf{H}_{1}$ (it is always possible since the multinorm is majorant). Then

$$
\theta_{F}\left(p, q_{0}, t\right)=\sup _{\|x\|_{q_{0}}^{\prime} \neq 0}\left\{\frac{\|F(t)(x)\|_{p}}{\|x\|_{q_{0}}^{\prime}}\right\} \leq \sup _{\|x\|_{q_{0}}^{\prime} \neq 0}\left\{\frac{C_{p}\|x\|_{q_{p}}^{\prime}}{\|x\|_{q_{0}}^{\prime}}\right\}=\tilde{C}_{p}\left(q_{0}\right),|t| \leq r .
$$

Thus, for each $r>0$ and each $p \in \mathcal{P}$ there exists $q_{0} \in \mathcal{Q}$ such that $\theta_{F}\left(p, q_{0}, t\right)$ (as functions of $t$ ) are bounded in the disk $|t| \leq r$. And it means that

$$
\forall r \forall p \exists q_{0}(p, r): M_{F}\left(p, q_{0}, r\right)<\infty .
$$

Hence, by Theorem 2 ,

$$
\varlimsup_{n \rightarrow \infty} \sqrt[n]{\theta_{\mathcal{A}}\left(p, q_{0}, n\right)} \leq \frac{1}{r}, r>0 .
$$

It follows from $(8)$ that either $\beta_{p}(\mathcal{A})<0$ or $\beta_{p}(\mathcal{A})=0$, but then by the arbitrariness of $r$

$$
\alpha_{p}(\mathcal{A})=\inf _{q \in \mathcal{Q}} \varlimsup_{n \rightarrow \infty} \sqrt[n]{\theta_{\mathcal{A}}(p, q, n)}=0 .
$$

Thus, the sequence $\left\{A_{n}\right\}$ belongs to the class $\mathfrak{L}_{\mathbf{H}_{1}, \mathbf{H}}[0,0]$ and therefore series (6) everywhere converges to the function $F(t)$ w.r.t. bornology (see [1, [5]).

Theorem 3 (Analogue of Liouville theorem). Suppose function (6) is entire and satisfies the condition

$$
\exists k \forall p \exists K_{p}>0 \exists q(p) \forall r>0: M_{F}(p, q, r) \leq K_{p} r^{k} .
$$

Then $F$ is an operator-valued polynomial of degree at most $k$, i.e.,

$$
F(t)=\sum_{n=0}^{[k]} A_{n} t^{n}
$$

Proof. By inequalities (7), (9) and the definition of the numbers $\theta_{\mathcal{A}}(p, q, n)$ we have

$$
\left\|A_{n}(x)\right\|_{p} \leq \theta_{\mathcal{A}}(p, q, n)\|x\|_{q}^{\prime} \leq K_{p} r^{k-n}\|x\|_{q}^{\prime}, \forall p \forall x \in \mathbf{H}_{1} \forall r>0 \forall n, q=q(p) .
$$

By the arbitrariness of $r$,

$$
\left\|A_{n}(x)\right\|_{p}=0, \forall n>k \forall p \forall x \in \mathbf{H}_{1}
$$

thus, $A_{n}=0, \forall n>k$.

Theorem 3 shows that if $F$ is an entire transcendental function, then the quantities $M_{F}(p, q, r)$ grows faster than any positive power as $r \rightarrow \infty$. 


\section{GROWTH CHARACTERISTICS FOR ENTIRE FUNCTION AND FORMULAE FOR THEIR CALCULATION}

Definition 6. Let $F: \mathbb{C} \rightarrow \operatorname{Lec}\left(\mathbf{H}_{1}, \mathbf{H}\right)$ be an entire transcendental function. The number $\rho_{p}(F)=\inf _{q \in \mathcal{Q}} \rho_{p, q}(F)$, where

$$
\rho_{p, q}(F)=\varlimsup_{r \rightarrow \infty} \frac{\ln \ln M_{F}(p, q, r)}{\ln r}
$$

will be called a p-order of the function $F$, and the number $\rho(F)=\sup _{p \in \mathcal{P}}\left\{\rho_{p}(F)\right\}$ will be called its order.

$$
\begin{aligned}
& \text { If } 0<\rho_{p}(F)<\infty \text {, the number } \sigma_{p}(F)=\inf _{q \in \mathcal{Q}} \sigma_{p, q}(F) \text {, where } \\
& \sigma_{p, q}(F)=\varlimsup_{r \rightarrow \infty} \frac{\ln M_{F}(p, q, r)}{r^{\rho_{p}(F)}},
\end{aligned}
$$

will be called a p-type of the function $f$ at p-order $\rho(F)$.

It can be shown that the case when for some $p, \rho_{p}(F)<\rho(F)$, while for other $\rho_{p}(F)=\rho(F)$, is reduced to the case $\rho_{p}(F)=\rho(F), \forall p$ by the replacement of the multinorm to an equivalent one. This is why (without loss of generality) we shall consider two cases, either $\rho_{p}(F)<\rho(F), \forall p$, or $\rho_{p}(F)=\rho(F), \forall p$.

Definition 7. Suppose a function $F(t)$ has p-orders $\rho_{p}(F)$ and order $0<\rho(F)<\infty$. The number

$$
\sigma(F)=\left\{\begin{array}{ccc}
0 & , & \rho_{p}(F)<\rho(F), \forall p \\
\sup _{p \in \mathcal{P}}\left\{\sigma_{p}(F)\right\} & , \quad \rho_{p}(F)=\rho(F), \forall p
\end{array}\right.
$$

will be called a type of the function $f$ at the order $\rho(F)$.

Lemma 1. Suppose

$$
\forall p \exists q_{p} \exists a_{p}, b_{p}>0 \exists r_{0}(p) \forall r>r_{0}: M_{F}\left(p, q_{p}, r\right)<e^{a_{p} r^{b_{p}}} .
$$

Then

$$
\forall p \exists n_{0}(p) \forall n>n_{0}: \sqrt[n]{\theta_{\mathcal{A}}\left(p, q_{p}, n\right)}<\left(\frac{a_{p} b_{p} e}{n}\right)^{\frac{1}{b_{p}}} .
$$

Proof. Suppose inequality (10) holds true, then by (7) we have

$$
\theta_{\mathcal{A}}\left(p, q_{p}, n\right)<\frac{e^{a_{p} r^{b_{p}}}}{r^{n}} ; \forall p \forall r>r_{0}(p) \forall n .
$$

We denote $\mu_{p}(r)=e^{a_{p} r^{k p}} r^{-n}$. It is obvious that

$$
\forall p: \mu_{p}(0)=\mu_{p}(+\infty)=+\infty
$$

Let us find $\min _{r>0}\left\{\mu_{p}(r)\right\}$,

$$
\begin{gathered}
\mu_{p}^{\prime}(r)=\mu_{p}(r) \ln ^{\prime} \mu_{p}(r) \\
\mu_{p}^{\prime}(r)=\mu_{p}(r)\left(a_{p} r^{b_{p}}-n \ln r\right)^{\prime} \\
\mu_{p}^{\prime}(r)=\mu_{p}(r)\left(a_{p} b_{p} r^{b_{p}-1}-\frac{n}{r}\right)
\end{gathered}
$$

$\mu_{p}^{\prime}(r)=0$ as $r=r_{1}=\left(\frac{n}{a_{p} b_{p}}\right)^{\frac{1}{b_{p}}}$. Substituting $r_{1}$ in inequality 12 , we obtain 11 . 
Lemma 2. Suppose

$$
\forall p \exists q_{p} \exists a_{p}, b_{p}>0 \exists n_{0}(p) \forall n>n_{0}: \sqrt[n]{\theta_{\mathcal{A}}\left(p, q_{p}, n\right)}<\left(\frac{a_{p} b_{p} e}{n}\right)^{\frac{1}{b_{p}}} .
$$

Then

$$
\forall p \forall \varepsilon>0 \exists r_{0}(p, \varepsilon) \forall r>r_{0}: M_{F}\left(p, q_{p}, r\right)<e^{\left(a_{p}+\varepsilon\right) r^{b_{p}}} .
$$

Proof. By condition $(13) \mathcal{A} \in \mathfrak{L}_{\mathbf{H}_{1}, \mathbf{H}}[0,0]$, thus, $F$ is an entire operator-valued function. Let us fix an arbitrary $p$ (and fix by this depending on it $q_{p}, a_{p}, b_{p}$ ) and consider the inequality

$$
\theta_{\mathcal{A}}\left(p, q_{p}, n\right) r^{n}<\left(\left(\frac{a_{p} b_{p} e}{n}\right)^{\frac{1}{b_{p}}} r\right)^{n}
$$

For sufficiently large $n$

$$
\left(\frac{a_{p} b_{p} e}{n}\right)^{\frac{1}{b_{p}}} r<\frac{1}{2}
$$

By $N_{p}(r)$ we denote the lowest of natural numbers $n$ for which inequality (15) holds true.

Let us find the dependence of $N_{p}(r)$ on $r$. We have

$$
2 r\left(\frac{a_{p} b_{p} e}{n}\right)^{\frac{1}{b_{p}}}<1 \text {, as } n>(2 r)^{b_{p}}\left(a_{p} b_{p} e\right) .
$$

Therefore, we can let $N_{p}(r)=\left[(2 r)^{b_{p}}\left(a_{p} b_{p} e\right)\right]+1$.

Further, for each fixed $p \in \mathcal{P}, t \in \mathbb{C}$ and $x \in \mathbf{H}_{1}$ we have

$$
\|F(t)(x)\|_{p} \leq \sum_{n=0}^{\infty}\left\|A_{n}(x)\right\|_{p}|t|^{n} \leq \sum_{n=0}^{\infty} \theta_{\mathcal{A}}\left(p, q_{p}, n\right)|t|^{n}\|x\|_{q_{p}}^{\prime},
$$

hence,

$$
\theta_{F}\left(p, q_{p}, t\right) \leq \sum_{n=0}^{\infty} \theta_{\mathcal{A}}\left(p, q_{p}, n\right)|t|^{n}
$$

i.e.,

$$
\forall p \forall r>0: M_{F}\left(p, q_{p}, r\right) \leq \sum_{n=0}^{\infty} \theta_{\mathcal{A}}\left(p, q_{p}, n\right) r^{n}=\sum_{n=0}^{N_{p}(r)-1} \theta_{\mathcal{A}}\left(p, q_{p}, n\right) r^{n}+\sum_{n=N_{p}(r)}^{\infty} \theta_{\mathcal{A}}\left(p, q_{p}, n\right) r^{n} .
$$

For $n \geq N_{p}(r)$ the inequality $\theta_{\mathcal{A}}\left(p, q_{p}, n\right) r^{n}<\left(\frac{1}{2}\right)^{n}$ holds true and hence

$$
\sum_{n=N_{p}(r)}^{\infty} \theta_{\mathcal{A}}\left(p, q_{p}, n\right) r^{n}<\sum_{n=N_{p}(r)}^{\infty}\left(\frac{1}{2}\right)^{n}<\sum_{n=0}^{\infty}\left(\frac{1}{2}\right)^{n}=2 .
$$

Since for each fixed $p$ and $r$

$$
\lim _{n \rightarrow \infty} \theta_{\mathcal{A}}\left(p, q_{p}, n\right) r^{n}=0
$$

the sequence $\left\{\theta_{\mathcal{A}}\left(p, q_{p}, n\right) r^{n}\right\}$ has a maximal term. Let

$$
m_{p}(r)=\max _{n \geq 0}\left\{\theta_{\mathcal{A}}\left(p, q_{p}, n\right) r^{n}\right\}
$$

then

$$
\sum_{n=0}^{N_{p}(r)-1} \theta_{\mathcal{A}}\left(p, q_{p}, n\right) r^{n} \leq m_{p}(r) N_{p}(r)
$$

Let us estimate $m_{p}(r)$. Let $\theta_{\mathcal{A}}\left(p, q_{p}, s\right) r^{s}$ be a maximal term. Under an unbound increasing of $r$ the index $s$ of maximal term increases unboundedly as well, i.e., $s \rightarrow \infty$ as $r \rightarrow \infty$. If $r$ is sufficiently large, then $s>n_{0}$, where $n_{0}$ is a number in (13). 
This is why

$$
m_{p}(r)=\theta_{\mathcal{A}}\left(p, q_{p}, s\right) r^{s}<\left(\frac{a_{p} b_{p} e}{s}\right)^{\frac{s}{b_{p}}} r^{s} \leq \max _{\xi \geq 0}\left\{\left(\frac{a_{p} b_{p} e}{\xi}\right)^{\frac{\xi}{b_{p}}} r^{\xi}\right\} .
$$

We denote

$$
\nu_{p}(\xi)=\left(\frac{a_{p} b_{p} e}{\xi}\right)^{\frac{\xi}{b_{p}}} r^{\xi}
$$

Clearly,

$$
\forall p: \nu_{p}(0)=1, \nu_{p}(+\infty)=0 .
$$

Let us find $\max _{\xi \geq 0}\left\{\nu_{p}(\xi)\right\}$. We have

$$
\nu_{p}^{\prime}(\xi)=\nu_{p}(\xi)\left(\frac{\ln \left(a_{p} b_{p} e\right)}{b_{p}}-\frac{\ln \xi}{b_{p}}-\frac{1}{b_{p}}+\ln r\right) .
$$

$\nu_{p}^{\prime}(\xi)=0$ as $\xi=\xi_{1}=\left(a_{p} b_{p}\right) r^{b_{p}}$.

$$
\nu_{p}\left(\xi_{1}\right)=e^{a_{p} r^{b_{p}}} .
$$

Therefore (for sufficiently large $r$ ), $m_{p}(r)<e^{a_{p} r^{b_{p}}}$.

Thus,

$$
M_{F}\left(p, q_{p}, r\right) \leq N_{p}(r) m_{p}(r)+2 \leq\left((2 r)^{b_{p}}\left(a_{p} b_{p} e\right)+1\right) e^{a_{p} r^{b_{p}}}+2<e^{\left(a_{p}+\varepsilon\right) r^{b_{p}}} .
$$

Theorem 4. The growth characteristics of function (6) are calculated by the formulae

$$
\begin{gathered}
\rho_{p}(F)=-\frac{1}{\beta_{p}(\mathcal{A})}, \forall p, \\
\sigma_{p}(F)=-\frac{\beta_{p}(\mathcal{A})}{e}\left(\alpha_{p}(\mathcal{A})\right)^{-\frac{1}{\beta_{p}(\mathcal{A})}}, \forall p, \\
\rho(F)=-\frac{1}{\beta(\mathcal{A})}, \\
\sigma(F)=\left\{\begin{array}{cc}
0 \quad & \beta_{p}(\mathcal{A})<\beta(\mathcal{A}), \forall p \\
-\frac{\beta(\mathcal{A})}{e}(\alpha(\mathcal{A}))^{-\frac{1}{\beta(\mathcal{A})}}, & \beta_{p}(\mathcal{A})=\beta(\mathcal{A}), \forall p .
\end{array}\right.
\end{gathered}
$$

Proof. We fix an arbitrary $p$. Suppose the $p$-order of the function $F$ equals $\rho_{p}(F)$. Then

$$
\forall p \forall \varepsilon>0 \exists q_{p}(\varepsilon) \exists r_{0}(p, \varepsilon) \forall r>r_{0}: M_{F}\left(p, q_{p}, r\right) \leq \exp \left\{r^{\rho_{p}(F)+\varepsilon}\right\} .
$$

By Lemma $1\left(b_{p}=\rho_{p}(F)+\varepsilon, a_{p}=1\right)$

$$
\sqrt[n]{\theta_{\mathcal{A}}\left(p, q_{p}, n\right)}<\left(\frac{\left(\rho_{p}(F)+\varepsilon\right) e}{n}\right)^{\frac{1}{\rho_{p}(F)+\varepsilon}}, \forall n>n_{0} .
$$

By this we successively find

$$
\begin{gathered}
\frac{1}{n} \ln \theta_{\mathcal{A}}\left(p, q_{p}, n\right)<\left(\frac{1}{\rho_{p}(F)+\varepsilon}\right) \ln \left(\left(\rho_{p}(F)+\varepsilon\right) e\right)-\frac{\ln n}{\rho_{p}(F)+\varepsilon}=C_{p}(\varepsilon)-\frac{\ln n}{\rho_{p}(F)+\varepsilon}, \\
\ln \theta_{\mathcal{A}}\left(p, q_{p}, n\right)<C_{p}(\varepsilon) n-\frac{n \ln n}{\rho_{p}(F)+\varepsilon}, \\
\ln \frac{1}{\theta_{\mathcal{A}}\left(p, q_{p}, n\right)}>\frac{n \ln n}{\rho_{p}(F)+\varepsilon}-C_{p}(\varepsilon) n=n \ln n\left(\frac{1}{\rho_{p}(F)+\varepsilon}-\frac{C_{p}(\varepsilon)}{\ln n}\right), \forall n>n_{0} .
\end{gathered}
$$


As $n \rightarrow \infty$, the expression in parentheses in 20 tends to $\frac{1}{\rho_{p}(F)+\varepsilon}$, and for large $n$

$$
\ln \frac{1}{\theta_{\mathcal{A}}\left(p, q_{p}, n\right)}>\frac{n \ln n}{\rho_{p}(F)+2 \varepsilon},
$$

i.e.,

By the arbitrariness of $\varepsilon$,

$$
\rho_{p}(F)+2 \varepsilon>\frac{n \ln n}{-\ln \theta_{\mathcal{A}}\left(p, q_{p}, n\right)} .
$$

$$
-\frac{1}{\beta_{p, q_{p}}(\mathcal{A})}=\varlimsup_{n \rightarrow \infty} \frac{n \ln n}{-\ln \theta_{\mathcal{A}}\left(p, q_{p}, n\right)} \leq \rho_{p}(F) .
$$

Since $\beta_{p}(\mathcal{A})=\inf _{q}\left\{\beta_{p, q}(\mathcal{A})\right\}$, then

$$
-\frac{1}{\beta_{p}(\mathcal{A})} \leq-\frac{1}{\beta_{p, q_{p}}(\mathcal{A})} \leq \rho_{p}(F)
$$

Hence, $\rho_{p}(F) \geq-\frac{1}{\beta_{p}(\mathcal{A})}, \forall p$.

Vice-versa, since

$$
-\frac{1}{\beta_{p, q}(\mathcal{A})}=\varlimsup_{n \rightarrow \infty} \frac{n \ln n}{-\ln \theta_{\mathcal{A}}(p, q, n)}
$$

then

$$
\frac{n \ln n}{-\ln \theta_{\mathcal{A}}(p, q, n)}<-\frac{1}{\beta_{p, q}(\mathcal{A})}+\frac{\varepsilon}{2}, \forall p \forall \varepsilon>0 \forall q \forall n>n_{0}(p, q, \varepsilon) .
$$

And since $\beta_{p}(\mathcal{A})=\inf _{q}\left\{\beta_{p, q}(\mathcal{A})\right\}$, then

$$
\forall p \forall \varepsilon>0 \exists q_{p}(\varepsilon):-\frac{1}{\beta_{p, q_{p}}(\mathcal{A})} \leq-\frac{1}{\beta_{p}(\mathcal{A})}+\frac{\varepsilon}{2} .
$$

Thus,

therefore,

$$
\forall p \forall \varepsilon>0 \exists q_{p}(\varepsilon) \exists n_{0}(p, \varepsilon) \forall n>n_{0}: \frac{n \ln n}{-\ln \theta_{\mathcal{A}}\left(p, q_{p}, n\right)}<-\frac{1}{\beta_{p}(\mathcal{A})}+\varepsilon
$$

$$
\begin{gathered}
\forall p \forall \varepsilon>0 \exists q_{p}(\varepsilon) \exists n_{0}(p, \varepsilon) \forall n>n_{0}: \sqrt[n]{\theta_{\mathcal{A}}\left(p, q_{p}, n\right)}<n^{-\frac{1}{-\frac{1}{\beta_{p}(\mathcal{A})}+\varepsilon}} . \\
\text { By Lemma } 2\left[\left(b_{p}=-\frac{1}{\beta_{p}(\mathcal{A})}+\varepsilon, a_{p}=\frac{1}{e\left(-\frac{1}{\beta_{p}(\mathcal{A})}+\varepsilon\right)}\right)\right. \\
\forall p \forall \varepsilon>0 \exists q_{p}(\varepsilon) \exists r_{0}(p, \varepsilon) \forall r>r_{0}: M_{F}\left(p, q_{p}, r\right) \leq \exp \left\{\left(a_{p}+\varepsilon\right) r^{\left(-\frac{1}{\beta_{p}(\mathcal{A})}+\varepsilon\right)}\right\} .
\end{gathered}
$$

It means that $\rho_{p}(F) \leq-\frac{1}{\beta_{p}(\mathcal{A})}, \forall p$.

Thus, identity (16) is proven. Identity (18) follows immediately from (16).

Let us prove identity (17).

Suppose the function $F$ has the $p$-order $0<\rho_{p}(F)<\infty$ and the $p$-type $\sigma_{p}(F)$. Then

$$
\forall p \forall \varepsilon>0 \exists q_{p}(\varepsilon) \exists r_{0}(p, \varepsilon) \forall r>r_{0}: M_{F}\left(p, q_{p}, r\right)<\exp \left\{\left(\sigma_{p}(F)+\varepsilon\right) r^{\rho_{p}(F)}\right\} .
$$

By Lemma 1 $\left(a_{p}=\sigma_{p}(F)+\varepsilon, b_{p}=\rho_{p}(F)\right)$ we have

$$
\begin{gathered}
\sqrt[n]{\theta_{\mathcal{A}}\left(p, q_{p}, n\right)}<\left(\frac{\left(\sigma_{p}(F)+\varepsilon\right) \rho_{p}(F) e}{n}\right)^{\frac{1}{\rho_{p}(F)}}, \forall n>n_{0}, \\
n^{\frac{1}{\rho_{p}(F)}} \sqrt[n]{\theta_{\mathcal{A}}\left(p, q_{p}, n\right)}<\left(\left(\sigma_{p}(F)+\varepsilon\right) \rho_{p}(F) e\right)^{\frac{1}{\rho_{p}(F)}}, \forall n>n_{0} .
\end{gathered}
$$


By the arbitrariness of $\varepsilon$

$$
\begin{gathered}
\alpha_{p, q_{p}}(\mathcal{A})=\varlimsup_{n \rightarrow \infty} n^{-\beta_{p}(\mathcal{A})} \sqrt[n]{\theta_{\mathcal{A}}\left(p, q_{p}, n\right)}= \\
=\varlimsup_{n \rightarrow \infty} n^{\frac{1}{\rho_{p}(F)}} \sqrt[n]{\theta_{\mathcal{A}}\left(p, q_{p}, n\right)} \leq\left(\sigma_{p}(F) \rho_{p}(F) e\right)^{\frac{1}{\rho_{p}(F)}}
\end{gathered}
$$

Since $\alpha_{p}(\mathcal{A})=\inf _{q}\left\{\alpha_{p, q}(\mathcal{A})\right\}$, then

$$
\alpha_{p}(\mathcal{A}) \leq \alpha_{p, q_{p}}(\mathcal{A}) \leq\left(\sigma_{p}(F) \rho_{p}(F) e\right)^{\frac{1}{\rho_{p}(F)}}, \forall p
$$

Vice-versa, since

$$
\alpha_{p, q}(\mathcal{A})=\varlimsup_{n \rightarrow \infty} n^{-\beta_{p}(\mathcal{A})} \sqrt[n]{\theta_{\mathcal{A}}(p, q, n)}=\varlimsup_{n \rightarrow \infty} n^{\frac{1}{\rho_{p}(F)}} \sqrt[n]{\theta_{\mathcal{A}}(p, q, n)}, \forall p, \quad \forall q
$$

then

$$
\begin{aligned}
\forall \varepsilon>0 \forall p \exists q(p, \varepsilon) \exists & n_{0}(p, \varepsilon) \forall n>n_{0}, \\
& \sqrt[n]{\theta_{\mathcal{A}}(p, q, n)}<\left(\frac{\left(\alpha_{p, q}(\mathcal{A})+\varepsilon\right)^{\rho_{p}(F)}}{n}\right)^{\frac{1}{\rho_{p}(F)}}<\left(\frac{\left(\alpha_{p}(\mathcal{A})+2 \varepsilon\right)^{\rho_{p}(F)}}{n}\right)^{\frac{1}{\rho_{p}(F)}} .
\end{aligned}
$$

By Lemma2 $2\left(b_{p}=\rho_{p}(F), a_{p}=\frac{\left(\alpha_{p}(\mathcal{A})+2 \varepsilon\right)^{\rho_{p}(F)}}{\rho_{p}(F) e}\right)$ we obtain

$$
\forall p \forall \varepsilon>0 \exists q_{p}(\varepsilon) \exists r_{0}(p, \varepsilon) \forall r>r_{0}: M_{F}\left(p, q_{p}, r\right)<\exp \left\{\left(a_{p}+\varepsilon\right) r^{\rho_{p}(F)}\right\} .
$$

It implies

By the arbitrariness of $\varepsilon$

$$
\sigma_{p}(F) \leq a_{p}=\frac{\left(\alpha_{p}(\mathcal{A})+2 \varepsilon\right)^{\rho_{p}(F)}}{\rho_{p}(F) e}
$$

$$
\sigma_{p}(F) \rho_{p}(F) e \leq\left(\alpha_{p}(\mathcal{A})\right)^{\rho_{p}(F)}
$$

therefore,

$$
\alpha_{p}(\mathcal{A}) \geq\left(\sigma_{p}(F) \rho_{p}(F) e\right)^{\frac{1}{\rho_{p}(F)}}
$$

i.e.,

$$
\sigma_{p}(F)=-\frac{\beta_{p}(\mathcal{A})}{e}\left(\alpha_{p}(\mathcal{A})\right)^{-\frac{1}{\beta_{p}(\mathcal{A})}}, \forall p .
$$

Hence, identity (17) is proven.

Let us prove identity (19).

If $\beta_{p}(\mathcal{A})<\beta(\mathcal{A}), \forall p$, from identity (16) it follows $\rho_{p}(F)<\rho(F), \forall p$ and by the definition $\sigma(F)=0$.

If $\beta_{p}(\mathcal{A})=\beta(\mathcal{A}), \forall p$, identity (16) yields $\rho_{p}(F)=\rho(F), \forall p$ and by the definition

$$
\sigma(F)=\sup _{p}\left\{\sigma_{p}(F)\right\}=-\frac{\beta(\mathcal{A})}{e} \sup _{p}\left\{\left(\alpha_{p}(\mathcal{A})\right)^{-\frac{1}{\beta(\mathcal{A})}}\right\}=-\frac{\beta(\mathcal{A})}{e}(\alpha(\mathcal{A}))^{-\frac{1}{\beta(\mathcal{A})}} .
$$

Remark. We observe that relation (16) is true also for $\rho_{p}(F)=\infty$. If we suppose $\rho_{p}(F)=$ $\infty$ and $\beta_{p}(\mathcal{A})<0$, by (above proven) $\rho_{p}(F)<\infty$ that is false. Similarly, identity (17) holds also for $\sigma_{p}(F)=\infty$.

Remark. Formulae (16) and (17) show that $p$-orders and $p$-type of an entire operator-valued function are completely determined by the characteristics of the sequence of its coefficients.

Examples. 
1. Let $\mathbf{H}_{1}=\mathbf{H}=\mathbf{H}(\mathbb{C})$ be the space of all entire functions with the topology of uniform convergence on the compacts

$$
\|x(z)\|_{p}=\max _{|z| \leq p}|x(z)|, p>0 .
$$

Let us find the characteristics of the function

$$
F(t)=e^{t \frac{d}{d z}}=\sum_{n=0}^{\infty} \frac{t^{n}}{n !} \frac{d^{n}}{d z^{n}}: \mathbb{C} \rightarrow \operatorname{Lec}(\mathbf{H}(\mathbb{C})) .
$$

The sequence $\mathcal{A}=\left\{\frac{1}{n !} \frac{d^{n}}{d z^{n}}\right\}$ has the following characteristics [1],

$$
\beta_{p}(\mathcal{A})=\alpha_{p}(\mathcal{A})=0, \forall p .
$$

Therefore, $\rho_{p}(F)=\infty, \forall p$.

2. Let $\mathbf{H}_{1}=[\rho, \sigma], \mathbf{H}=[\rho, \theta], \theta \geq \sigma$. The topologies on these spaces are determined by the multinorms

$$
\begin{gathered}
\|x(z)\|_{\varepsilon}=\sup _{p>0}\left\{\max _{|z| \leq p}|x(z)| e^{-(\sigma+\varepsilon) p^{\rho}}\right\}, \varepsilon>0, x \in[\rho, \sigma] . \\
\|y(z)\|_{\varepsilon}=\sup _{p>0}\left\{\max _{|z| \leq p}|y(z)| e^{-(\theta+\varepsilon) p^{\rho}}\right\}, \varepsilon>0, y \in[\rho, \theta] .
\end{gathered}
$$

Let us find the characteristics of the function

$$
F(t)=e^{t \frac{d}{d z}}=\sum_{n=0}^{\infty} \frac{t^{n}}{n !} \frac{d^{n}}{d z^{n}}: \mathbb{C} \rightarrow \operatorname{Lec}([\rho, \sigma],[\rho, \theta]) .
$$

The sequence $\mathcal{A}=\left\{\frac{1}{n !} \frac{d^{n}}{d z^{n}}\right\}$ has the following characteristics [1]:

$$
\beta_{\varepsilon}(\mathcal{A})=-\frac{1}{\rho}, \alpha_{\varepsilon}(\mathcal{A})=\left(\rho e \sigma \Omega_{\varepsilon}\right)^{\frac{1}{\rho}}, \forall \varepsilon
$$

where

$$
\Omega_{\varepsilon}=\left\{\begin{array}{cc}
\left(1-\left(\frac{\sigma}{\theta+\varepsilon}\right)^{\frac{1}{\rho-1}}\right)^{1-\rho} & , \quad \rho>1 \\
1 & , \quad \rho \leq 1
\end{array}\right.
$$

Therefore,

$$
\rho_{\varepsilon}(F)=\rho, \sigma_{\varepsilon}(F)=\sigma \Omega_{\varepsilon}, \forall \varepsilon
$$

3. Let $\mathbf{H}_{1}=\mathbf{H}=\mathbf{H}(\mathbb{C})$ be the space of all entire functions with the topology of uniform convergence on the compacts

$$
\|x(z)\|_{p}=\max _{|z| \leq p}|x(z)|, p>0 .
$$

Let us find the characteristics of the function

$$
\begin{aligned}
F(t)(x)=x(z)+t \int_{0}^{z} e^{(z-\xi) t} x(\xi) d \xi= & \\
& =x(z)+t \sum_{n=0}^{\infty} \int_{0}^{z} \frac{(z-\xi)^{n} t^{n}}{n !} x(\xi) d \xi,
\end{aligned}
$$$$
F(t): \mathbb{C} \rightarrow \operatorname{Lec}(\mathbf{H}(\mathbb{C}))
$$

Here

$$
A_{n}(x)=\int_{0}^{z} \frac{(z-\xi)^{n-1}}{(n-1) !} x(\xi) d \xi, n=1,2, \ldots, A_{0}=E
$$


The sequence $\mathcal{A}=\left\{A_{n}\right\}$ has the following characteristics [1],

$$
\beta_{p}(\mathcal{A})=-1, \alpha_{p}(\mathcal{A})=p, \forall p .
$$

Therefore, $\rho_{p}(F)=1, \sigma_{p}(F)=\frac{p}{e}, \forall p$.

\section{PROPERTIES OF GROWTH CHARACTERISTICS FOR OPERATOR-VALUED FUNCTIONS}

Let us note certain properties of the growth characteristics for operator-valued functions implied by Theorem 4 .

$1^{0}$. Entire function $F$ and its $k$ th derivative $F^{(k)}$ has the same $p$-orders and p-types of growth.

The validity follows from the fact the sequences $\left\{A_{n}\right\}$ and $\left\{\frac{(n+k) !}{n !} A_{n+k}\right\}$ has the same characteristics for each fixed $k$.

$2^{0}$. If a function $F_{1}$ has the p-orders $\rho_{p}\left(F_{1}\right)$ and the $p$-types $\sigma_{p}\left(F_{1}\right)$, and a function $F_{2}$ has the p-orders $\rho_{p}\left(F_{2}\right)>\rho_{p}\left(F_{1}\right), \forall p$ and the p-types $\sigma_{p}\left(F_{2}\right)$, the function $F=F_{1}+F_{2}$ has the $p$-orders $\rho_{p}(F)=\rho_{p}\left(F_{2}\right), \forall p$ and the $p$-types $\sigma_{p}(F)=\sigma_{p}\left(F_{2}\right), \forall p$.

The validity is implied by the fact that the characteristics of the sum of operators are equal to the characteristics of the term of the greater order.

$3^{0}$. If a function $F_{1}$ has the p-orders $\rho_{p}\left(F_{1}\right)$ and the p-types $\sigma_{p}\left(F_{1}\right)$, and a function $F_{2}$ has the p-orders $\rho_{p}\left(F_{2}\right)=\rho_{p}\left(F_{1}\right), \forall p$ and the p-types $\sigma_{p}\left(F_{2}\right)>\sigma_{p}\left(F_{1}\right), \forall p$, then the function $F=F_{1}+F_{2}$ has the $p$-orders $\rho_{p}(F)=\rho_{p}\left(F_{2}\right), \forall p$ and the $p$-types $\sigma_{p}(F)=\sigma_{p}\left(F_{2}\right), \forall p$.

The validity follows from the fact that the characteristics of the sum of operators of same orders are equal to the characteristics of the term of the greater type.

$4^{0}$. (The case $\mathbf{H}_{1}=\mathbf{H}$.) Suppose a function $F_{1}$ has the order $\rho\left(F_{1}\right)$ and the type $\sigma\left(F_{1}\right)$, and a function $F_{2}$ has the order $\rho\left(F_{2}\right)>\rho\left(F_{1}\right)$ and the type $\sigma\left(F_{2}\right)$. Then the function $F=F_{1} F_{2}$ has the order $\rho(F) \leq \rho\left(F_{2}\right)$ and the type $\sigma(F)$. If $\rho(F)=\rho\left(F_{2}\right)$, then $\sigma(F) \leq \sigma\left(F_{2}\right)$. A similar statement holds for the function $\tilde{F}=F_{2} F_{1}$.

The proof is based on the following lemma.

Lemma 3. Suppose a sequence of operators $\mathcal{A}=\left\{A_{n}\right\}$ has the order $\beta(\mathcal{A})$ and the type $\alpha(\mathcal{A})$, and a sequence of the operators $\mathcal{B}=\left\{B_{n}\right\}$ has the order $\beta(\mathcal{B})>\beta(\mathcal{A})$ and the type $\alpha(\mathcal{B})$. Then the sequence of the operators $\mathcal{C}=\left\{C_{n}\right\}$, where $C_{n}=\sum_{k=0}^{n} A_{k} B_{n-k}$ has the order $\beta(\mathcal{C}) \leq \beta(\mathcal{B})$ and the type $\alpha(\mathcal{C})$. If $\beta(\mathcal{C})=\beta(\mathcal{B})$, then $\alpha(\mathcal{C}) \leq \alpha(\mathcal{B})$.

Proof. Denote $a=\alpha(\mathcal{A}) e^{\beta(\mathcal{A})}, b=\alpha(\mathcal{B}) e^{\beta(\mathcal{B})}$.

The definition of the order and type of a sequence of operators implies [1]

$$
\begin{aligned}
& \forall \varepsilon, \varepsilon_{1}>0, \forall p, \exists M_{p}, \exists q, \forall n, \forall x \in \mathbf{H}: \\
& \left\|C_{n}(x)\right\|_{p} \leq M_{p}\left((b+\varepsilon)^{n} n !^{\beta(\mathcal{B})}+\left(a+\varepsilon_{1}\right)(b+\varepsilon)^{n-1} 1 !^{\beta(\mathcal{A})}(n-1) !^{\beta(\mathcal{B})}+\cdots+\right. \\
& \left.+\left(a+\varepsilon_{1}\right)^{n-1}(b+\varepsilon)(n-1) !^{\beta(\mathcal{A})} 1 !^{\beta(\mathcal{B})}+\left(a+\varepsilon_{1}\right)^{n} n !^{\beta(\mathcal{A})}\right)\|x\|_{q} \leq \\
& \leq M_{p}(b+\varepsilon)^{n} n !^{\beta(\mathcal{B})}\left[1+\left(\begin{array}{c}
n \\
1
\end{array}\right)^{-\beta(\mathcal{B})}\left(\frac{a+\varepsilon_{1}}{b+\varepsilon}\right) 1 !^{\nu}+\left(\begin{array}{c}
n \\
2
\end{array}\right)^{-\beta(\mathcal{B})}\left(\frac{a+\varepsilon_{1}}{b+\varepsilon}\right)^{2} 2 !^{\nu}+\cdots+\right. \\
& \left.\quad+\left(\begin{array}{c}
n \\
n
\end{array}\right)^{-\beta(\mathcal{B})}\left(\frac{a+\varepsilon_{1}}{b+\varepsilon}\right)^{n} n !^{\nu}\right]\|x\|_{q},
\end{aligned}
$$

where $\nu=\beta(\mathcal{A})-\beta(\mathcal{B})$. 
If $\beta(\mathcal{B})>\beta(\mathcal{A})(\nu<0)$, for large $n$ the expression in brackets in 21) does not exceed $\left(1+\varepsilon_{2}\right)^{n}, \forall \varepsilon_{2}>0$ and thus $\beta(\mathcal{C}) \leq \beta(\mathcal{B})$, and if $\beta(\mathcal{C})=\beta(\mathcal{B})$, then $\alpha(\mathcal{C}) \leq \alpha(\mathcal{B})$.

$5^{0}$. (The case $\mathbf{H}_{1}=\mathbf{H}$.) Suppose a function $F_{1}$ has the order $\rho\left(F_{1}\right)$ and the type $\sigma\left(F_{1}\right)$, and a function $F_{2}$ has the order $\rho\left(F_{2}\right)=\rho\left(F_{1}\right)$ and the type $\sigma\left(F_{2}\right) \geq \sigma\left(F_{1}\right)$. Then the function $F=F_{1} F_{2}$ has the order $\rho(F) \leq \rho\left(F_{2}\right)$ and the type $\sigma(F)$. If $\rho(F)=\rho\left(F_{2}\right)$, then $\sigma(F) \leq 2 \sigma\left(F_{2}\right)$. $A$ similar statement holds true for the function $\tilde{F}=F_{2} F_{1}$.

The proof is based on the following lemma.

Lemma 4. Let a sequence of operators $\mathcal{A}=\left\{A_{n}\right\}$ has the order $\beta(\mathcal{A})$ and the type $\alpha(\mathcal{A})$, and a sequence of operators $\mathcal{B}=\left\{B_{n}\right\}$ does the order $\beta(\mathcal{B})=\beta(\mathcal{A})$ and the type $\alpha(\mathcal{B}) \geq \alpha(\mathcal{A})$. The the sequence of the operators $\mathcal{C}=\left\{C_{n}\right\}$, where $C_{n}=\sum_{k=0}^{n} A_{k} B_{n-k}$, has the order $\beta(\mathcal{C}) \leq \beta(\mathcal{B})$ and the type $\alpha(\mathcal{C})$. If $\beta(\mathcal{C})=\beta(\mathcal{B})$, then $\alpha(\mathcal{C}) \leq 2^{-\beta(\mathcal{B})} \alpha(\mathcal{B})$.

Proof. Under the hypothesis of the lemma the expression in the brackets in (21) does not exceed $2^{-\beta(\mathcal{B}) n} n$ and thus $\beta(\mathcal{C}) \leq \beta(\mathcal{B})$, and if $\beta(\mathcal{C})=\beta(\mathcal{B})$, then $\alpha(\mathcal{C}) \leq 2^{-\beta(\mathcal{B})} \alpha(\mathcal{B})$.

Remark. As it is known, in the scalar case the theorem on categories [3, Th. 12] is valid. In the case of operator-valued function this question is still open.

$6^{0}$. (Invariance). Suppose $\mathbf{H}_{1}, \widetilde{\mathbf{H}}_{1}, \mathbf{H}$ and $\widetilde{\mathbf{H}}$ are four locally convex spaces with the topologies induced respectively by the multinorms $\|\cdot\|_{q}^{\prime}, q \in \mathcal{Q},\|\cdot\|_{\widetilde{q}}^{\prime}, \widetilde{q} \in \widetilde{\mathcal{Q}},\|\cdot\|_{p}, p \in \mathcal{P},\|\cdot\|_{\widetilde{p}}, \widetilde{p} \in \widetilde{\mathcal{P}}$ and let $T_{1}: \mathbf{H}_{1} \rightarrow \widetilde{\mathbf{H}}_{1}, T: \mathbf{H} \rightarrow \widetilde{\mathbf{H}}$ are two topological isomorphisms. Then

1) for each operator-valued function

$$
F(t)=\sum_{n=0}^{\infty} A_{n} t^{n}: \mathbb{C} \rightarrow \operatorname{Lec}\left(\mathbf{H}_{1}, \mathbf{H}\right)
$$

its order and type coincide with the order and type of the function

$$
\tilde{F}(t)=\sum_{n=0}^{\infty} T A_{n} T_{1}^{-1} t^{n}: \mathbb{C} \rightarrow \operatorname{Lec}\left(\widetilde{\mathbf{H}}_{1}, \widetilde{\mathbf{H}}\right) ;
$$

2 ) if all the $p$-orders of the function $F$ are strictly less than its order, then all the $\widetilde{p}$-orders of the function $\tilde{F}$ are strictly less than its order;

3 ) if at least one $p$-order of the function $F$ equals to its order, then at least one $\widetilde{p}$-order of the function $\tilde{F}$ equals to its order;

4 ) if the function $F$ has the $p$-orders $\rho_{p}(F)$, the order $\rho(F)$, the $p$-types $\sigma_{p}(F)$ and the type $\sigma(F)$, at that the set

$$
\mathcal{P}_{F}=\left\{p \in \mathcal{P}: \rho_{p}(F)=\rho(F)\right\}
$$

is non-empty and $\forall p \in \mathcal{P}_{F}: \sigma_{p}(F)<\sigma(F)$, then the function $\tilde{F}$ has the $\tilde{p}$-orders $\rho_{\tilde{p}}(\tilde{F})$, the order $\rho(\tilde{F})$, the $\widetilde{p}$-types $\sigma_{\widetilde{p}}(\tilde{F})$ and the type $\sigma(\tilde{F})$, at that the set

$$
\widetilde{\mathcal{P}}_{\tilde{F}}=\left\{\widetilde{p} \in \widetilde{\mathcal{P}}: \rho_{\widetilde{p}}(\tilde{F})=\rho(\tilde{F})\right\}
$$

is non-empty and $\forall \widetilde{p} \in \widetilde{\mathcal{P}}_{\tilde{F}}: \sigma_{\widetilde{p}}(\tilde{F})<\sigma(\tilde{F})$;

$5)$ if under hypothesis of Item 4) $\exists p \in \mathcal{P}_{F}: \sigma_{p}(F)=\sigma(F)$, then $\exists \widetilde{p} \in \widetilde{\mathcal{P}}_{\tilde{F}}: \sigma_{\tilde{p}}(\tilde{F})=\sigma(\tilde{F})$.

The validity of property $6^{0}$ follows from analogous properties for the characteristics of a sequence of operators [1, 6].

The invariance property implies that under any replacements of the multinorms in $\mathbf{H}_{1}$ and $\mathbf{H}$ to equivalent ones ( $T_{1}$ and $T$ are identity operators)

1 ) the order and type of the operator-valued function $F$ remain the same; 
2 ) if all the $p$-orders of the function $F$ were strictly less than its order before the replacement of the multinorms, after the replacement of the multinorms all its $\widetilde{p}$-orders are also strictly less than the order;

3 ) if at least one $p$-order of the function $F$ equals its order before the replacement of the multinorms, after the replacement at least one its $\widetilde{p}$-order (not necessarily the same) is also equal to its order;

4 ) if the function $F$ has the $p$-order $\rho_{p}(F)$ before the replacement of the multinorms, the order $\rho(F)$, the $p$-types $\sigma_{p}(F)$, and the type $\sigma(F)$, at that the set

$$
\mathcal{P}_{F}=\left\{p \in \mathcal{P}: \rho_{p}(F)=\rho(F)\right\}
$$

is non-empty and $\forall p \in \mathcal{P}_{F}: \sigma_{p}(F)<\sigma(F)$, then after the replacement of the multinorms this function has the $\widetilde{p}$-orders $\rho_{\widetilde{p}}(F)$, the order $\rho(F)$, the $\widetilde{p}$-types $\sigma_{\widetilde{p}}(F)$, and the type $\sigma(F)$, at that the set

$$
\widetilde{\mathcal{P}}_{F}=\left\{\widetilde{p} \in \widetilde{\mathcal{P}}: \rho_{\widetilde{p}}(F)=\rho(F)\right\}
$$

is non-empty and $\forall \widetilde{p} \in \widetilde{\mathcal{P}}_{F}: \sigma_{\widetilde{p}}(F)<\sigma(F)$;

5) if under the hypothesis of Item 4) $\exists p \in \mathcal{P}_{F}: \sigma_{p}(F)=\sigma(F)$, then $\exists \widetilde{p} \in \widetilde{\mathcal{P}}_{F}: \sigma_{\widetilde{p}}(F)=\sigma(F)$.

\section{BIBLIOGRAPHY}

1. V.P. Gromov, S.N. Mishin, S.V. Panyushkin. Operators of finite order and differential-operator equations. Orel State University, Orel. 2009. (in Russian).

2. L.V. Kantorovich, G.P. Akilov. Functional analysis in normed spaces. Fizmatgiz, Moscow. 1959. [International Series of Monographs in Pure and Applied Mathematics, V. 46. Pergamon Press, N.Y. 1964.]

3. B.Ya. Levin. Distribution of Zeros of Entire Functions. GITTL, Moscow. 1956. [American Mathematical Soc., Translations of mathematics monographs. V. 5. 1980.]

4. A.F. Leont'ev. Entire functions. Exponential series. Nauka, Moscow. 1983. (in Russian).

5. S.N. Mishin. Connection of the characteristics of sequence of the operators with convergence by bornology. // Vestnik RUDN. Ser. matem. informat. fiz. 2010. No. 4. P. 26-34. (in Russian).

6. S.N. Mishin. Invariance of characteristics of sequence of operators and operator characteristics of vector. // Uchenye zapiski OGU (lab. TFFA). 2010. No. 7. P. 32-35. (in Russian).

7. S.N. Mishin. Finite order operators in locally convex spaces and there applications. Dissertation for candidate of phys. and math. sci. degree. Orel. 2002.

8. Ya.V. Radyno. Linear equations and bornology. Belorussian State University, Minsk. 1982.

9. P. Garrett. Holomorphic vector-valued functions. Course notes. 2005. Electronic resource. URL: http://www.math.umn.edu/ garrett/m/fun/Notes/09_vv_holo.pdf

Sergei Nikolaevich Mishin,

Orel State University,

Komsomolskaya str., 95,

302026, Orel, Russia

E-mail: sergeymishin@rambler.ru 